Article

\title{
The Influence of Laser Linewidth on the Brillouin Shift Frequency Accuracy of BOTDR
}

\author{
Qing Bai ${ }^{1}{ }^{\oplus}$, Min Yan $^{1}{ }^{1}$, Bo Xue ${ }^{1}$, Yan Gao ${ }^{1}$, Dong Wang ${ }^{1}$, Yu Wang ${ }^{1}{ }^{\oplus}$, Mingjiang Zhang ${ }^{1}$, \\ Hongjuan Zhang ${ }^{1}$ and Baoquan Jin ${ }^{1,2, *(1)}$ \\ 1 Key Laboratory of Advanced Transducers and Intelligent Control Systems (Ministry of Education and \\ Shanxi Province), Taiyuan University of Technology, Taiyuan 030024, China; \\ baiqing0122@link.tyut.edu.cn (Q.B.); yanmin_tyut@163.com (M.Y.); xuebo0951@link.tyut.edu.cn (B.X.); \\ gaoyan@tyut.edu.cn (Y.G.); wangdong@tyut.edu.cn (D.W.); wangyu@tyut.edu.cn (Y.W.); \\ zhangmingjiang@tyut.edu.cn (M.Z.); zhanghongjuan@tyut.edu.cn (H.Z.) \\ 2 State Key Laboratory of Coal and CBM Co-mining, Shanxi Jincheng Anthracite Mining Group Co., Ltd., \\ Jincheng 048000, China \\ * Correspondence: jinbaoquan@tyut.edu.cn; Tel.: +86-138-3515-5702
}

Received: 9 December 2018; Accepted: 21 December 2018; Published: 25 December 2018

Featured Application: Brillouin-based distributed optical fiber sensing technology, such as BOTDR, BOTDA, BOCDR, BOCDA.

\begin{abstract}
This paper analyzes the influence of laser linewidth on the measurement accuracy of a frequency-scanning Brillouin optical time domain reflectometer (FS-BOTDR), allowing for both the width of Brillouin gain spectrum and the signal-to-noise ratio (SNR) of the BOTDR system. The measurement accuracy of the Brillouin frequency shift (BFS) is theoretically investigated versus the duration of the probe pulse and the linewidth of the laser source, by numerically simulating how a FS-BOTDR works and evaluating the Brillouin gain spectrum (BGS) width and the system SNR. The simulation results show that the BFS accuracy is improved as the laser linewidth becomes narrower when the probe pulse width is fixed. We utilize five types of lasers with respective linewidths of $1.05 \mathrm{MHz}, 101 \mathrm{kHz}, 10.2 \mathrm{kHz}, 3.1 \mathrm{kHz}$, and $98 \mathrm{~Hz}$ to compare the BFS measurement accuracy over a $\sim 10 \mathrm{~km}$ optical sensing fiber. The experimental results demonstrate that the root-mean-square error (RMSE) of BFS decreases with the laser linewidth narrowing from $1.05 \mathrm{MHz}$ to $3.1 \mathrm{kHz}$, which is in good agreement with the numerical simulation. However, the RMSE of BFS increases when the laser linewidth is less than $3.1 \mathrm{kHz}$, which may arise from the coherent Rayleigh noise due to a too narrow laser linewidth. The results can provide a theoretical basis and experimental guidance for choosing the appropriate laser linewidth in BOTDR.
\end{abstract}

Keywords: Distributed optical fiber sensing; BOTDR; measurement accuracy; laser linewidth; signal-to-noise ratio

\section{Introduction}

Brillouin optical time domain reflectometer (BOTDR) was firstly proposed as continuouslydistributed optical fiber sensing technology in 1993 [1]. In recent decades, it has attracted much attention due to its advantages of long-distance measurement, corrosion resistance, anti-electromagnetic interference, and one-end access especially [2-4]. Meanwhile, with the development of its capacity of simultaneous measurement of strain and temperature [5,6], BOTDR has been more and more widely utilized in many industrial applications, such as monitoring operation status and structural health conditions of transmission lines, soil slopes, large-scale bridges, gas/oil pipelines, transportation tunnels, 
and underground mines [7-10]. Consequently, the measurement accuracy of Brillouin frequency shift (BFS), which determines the temperature and strain accuracy, has inevitably been a focused issue for performance enhancement of BOTDR.

The measurement accuracy of BFS can be affected by numerous factors, such as the fitting algorithm for Brillouin gain spectrum (BGS), the features of probe pulses, and even the characteristics of the laser source. Hence, a large number of papers have focused on above factors to discuss and improve the accuracy of BOTDR. Originally, a simple Levenberg-Marquart algorithm was used for BGS fitting [11]. Then, a series of time-frequency analysis methods called Cohen's class were proposed for the signal processing of BOTDR, reducing the BFS fluctuation by three times [12]. For improving both the measurement accuracy and data processing speed, a similarity matching method was proposed, making the standard derivation of BFS results three times better [13]. An iterative quadratic fitting method can also be utilized to extract BFS from noisy signals for improvement of the BFS accuracy [14]. Besides, the features of probe light pulses also have a significant influence on the accuracy of BFS, including the pulse shape and pulse extinction ratio. Hao et al. analyzed the effects of different modulated pulse on the backscattered Brillouin power spectra of BOTDR, including the pulse shapes of Lorentzian, Gaussian, hyperbolic-secant, super Gaussian, triangular, and rectangular pulses $[15,16]$. The pulse sequences were complementarily coded in a BOTDR scheme to achieve a high measurement accuracy and fast measurement speed [17]. The simplex pulse codes were also testified to be beneficial for improving the signal-to-noise ratio ratio (SNR) of the BOTDR signal and the measurement accuracy of BFS [18]. The higher extinction ratio of the probe pulses was capable of enhancing the SNR as well as reducing the measurement uncertainty of BFS [19,20]. Moreover, it is noted that the wavelength and linewidth of the laser source likewise impact BFS accuracy. Lalam et al. used a wavelength diversity technique with a Brillouin ring laser in a conventional BOTDR system to improve SNR [21]. A multi-wavelength heterodyne-detection technique was utilized to provide $4.2 \mathrm{~dB}$ SNR enhancement, enabling the measurement accuracy to be increased by two times [22]. As an important parameter of the laser, the wide linewidth imposes a broadening effect on BGS, which affects the BFS accuracy partly, but the broadening effect is indistinctive when the laser linewidth is less than $1 \mathrm{MHz}$ [23]. Meanwhile, it has been proven that the BFS accuracy is determined not only by the width of BGS, but also by the SNR of the BOTDR system [24]. The system SNR of BOTDR is inherently related with the laser linewidth closely, because the coherent heterodyne detection, which is sensitive to the laser linewidth, is commonly used in BOTDR [25]. Hence, the influence of the laser linewidth on the BFS accuracy of BOTDR needs to be further discussed in detail, considering both the BGS width and the system SNR.

In this paper, how the BFS accuracy is determined by the linewidth of the laser source is theoretically analyzed and experimentally verified in a frequency-scanning BOTDR (FS-BOTDR). The broadening effect of BGS was analyzed, allowing for both the pulse width of the probe light and the frequency-scanning process in an FS-BOTDR. Then, the SNR of BOTDR with coherent heterodyne detection was calculated, treating the phase fluctuation as the primary noise source. The BFS accuracy was finally simulated numerically taking into account both the BGS and the SNR synthetically. We used five lasers with respective linewidths of $1.05 \mathrm{MHz}, 101 \mathrm{kHz}, 10.2 \mathrm{kHz}, 3.1 \mathrm{kHz}$, and $98 \mathrm{~Hz}$ to perform temperature measurement in the BOTDR sensing system and verify the numerical simulation. The results of this research will be helpful to choose the laser linewidth for the BOTDR.

\section{Numerical Simulation}

Figure 1 gives a classical schematic diagram of BOTDR with coherent heterodyne detection [26]. One branch of the seed laser is modulated by an optical modulator to generate probe pulse light, which is injected into the tested fiber through an optical circulator. The other one branch passing a polarization controller is injected into a photodetector to beat with the Brillouin backscattering. The output electronic signal is orderly down-converted by mixing with a frequency scanner, filtered by a band-pass filter (BPF), and acquired by a data acquisition (DAQ) digitalizer. 


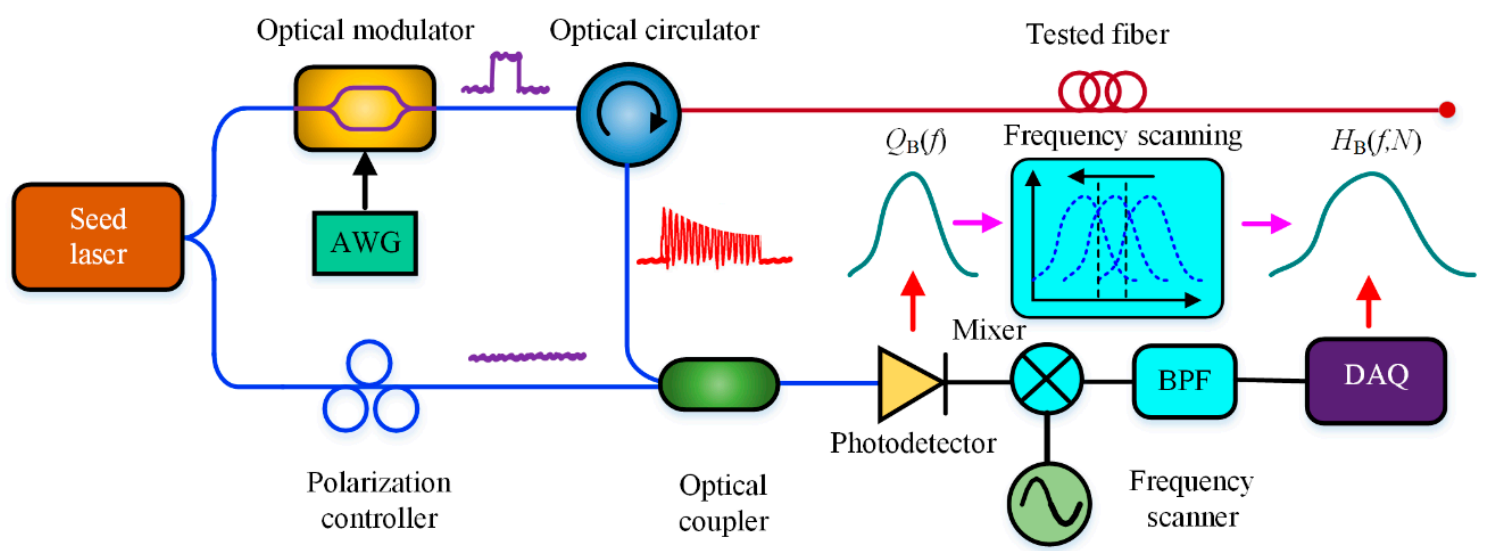

Figure 1. Schematic diagram of frequency-scanning BOTDR (FS-BOTDR) with coherent heterodyne detection: $\mathrm{AWG}=$ Arbitrary Waveform Generator; $\mathrm{BPF}=$ Band-pass Filter; $\mathrm{DAQ}=$ Data Acquisition.

The measurement error of BFS in FS-BOTDR is related with both the width of BGS denoted as $W_{\mathrm{BGS}}$ and the system SNR, which is given by Equation (1) [1]:

$$
\Delta \alpha=\frac{W_{\mathrm{BGS}}}{\sqrt{2}(S N R)^{1 / 4}}
$$

hence, the values of $W_{\text {BGS }}$ and $S N R$ need to be both analyzed for figuring out the BFS accuracy of $\Delta \alpha$, as specified below.

When the continuous lightwave is injected into the tested fiber, the obtained BGS presents a Lorentzian shape, given by [27]:

$$
G_{\mathrm{B}}(f)=g_{0} \frac{(w / 2)^{2}}{\left(f-f_{\mathrm{B}}\right)^{2}+(w / 2)^{2}}
$$

where $w$ is the full-width at half maximum (FWHM) of BGS, $f_{\mathrm{B}}$ is the Brillouin frequency shift, and $\mathrm{g}_{0}$ is the Brillouin gain coefficient, which is the BGS peak value when $f=f_{\mathrm{B}}$.

When the continuous lightwave is modulated into the pulse light with a peak power of $P_{0}$ and pulse width of $\tau$, the power spectrum of the pulse probe light can be expressed as:

$$
P_{\mathrm{p}}\left(f, f_{.0}\right)=P_{0}\left[\frac{\sin \pi\left(f-f_{0}\right) \tau}{\pi\left(f-f_{0}\right)}\right]^{2},
$$

where $f_{0}$ is the optical frequency of the probe light. Hence, the Brillouin backscattered-light power spectrum for pulse light can be calculated by [24]:

$$
Q_{\mathrm{B}}(f)=\int_{-\infty}^{+\infty} P_{\mathrm{p}}\left(f, f_{0}\right) G_{\mathrm{B}}(f)=\frac{g_{0} P_{0} \tau}{\eta^{2}+1}\left\{1+\frac{\eta^{2}-1-e^{-\tau w \pi}\left[\left(\eta^{2}-1\right) \cos \tau w \eta \pi+2 \sigma \sin \eta \tau w \pi\right]}{\tau w\left(\eta^{2}+1\right) \pi}\right\}
$$

where:

$$
\eta=\frac{f-f_{B}}{w / 2}
$$

In the FS-BOTDR, the power spectrum, $Q_{\mathrm{B}}(f)$, is always measured by the frequency-scanning method. When the frequency scanner is tuned at a certain frequency point, the integrated power of every frequency segment passing the BPF is acquired by DAQ. Hence, as the frequency scanner is tuned step by step, the final measured spectrum after frequency-scanning process, denoted as $H_{\mathrm{B}}(f, N)$, can be given by:

$$
H_{\mathrm{B}}(f, N)=\left\{Q_{\mathrm{B}}^{1}(f), Q_{\mathrm{B}}^{2}(f), \ldots, Q_{\mathrm{B}}^{\mathrm{N}}(f)\right\}
$$




$$
Q_{\mathrm{B}}^{i}(f)=\int_{f_{s}+(i-1) f_{\text {step }}-B / 2}^{f_{\mathrm{s}}+(i-1) f_{\text {step }}+B / 2} Q_{\mathrm{B}}(f) d f, i=1,2, \ldots, N .
$$

where $f_{\mathrm{s}}$ and $f_{\text {step }}$ are, respectively, the start frequency and frequency step during the frequency-scanning process, $N$ is the number of frequency points, and $B$ is the bandwidth of the BPF. Based on Equation (4) Equation (7), the broadening effect of BGS in FS-BOTDR is numerically simulated. The model parameters utilized for simulations are listed in Table A1 of Appendix A. The simulation results are shown in Figure 2. It can been clearly seen that the finally measured BGS width in FS-BOTDR, denoted as $W_{\mathrm{BGS}}$, is larger than both the original BGS width for continuous light and the BGS width for pulse light. Hence, the broadening effect of BGS in FS-BOTDR must be taken into account when the BFS accuracy is evaluated according to Equation (2).



Figure 2. Numerical simulation of the broadening effect of Brillouin gain spectrum (BGS) in FS-BOTDR: $G_{\mathrm{B}}(f)$ is the original BGS for the continuous light; $Q_{\mathrm{B}}(f)$ is the BGS for the pulse light with a width of $22 \mathrm{~ns} ; H_{\mathrm{B}}(f, N)$ is the final measured BGS through the frequency-scanning process when the width of the probe pulse is $22 \mathrm{~ns}$.

Figure 3a gives profiles of $H_{\mathrm{B}}(f, N)$ under different probe pulse widths. The peak power is normalized by that of the spectrum profile obtained when the pulse width is $12 \mathrm{~ns}$. The spectrum width and relative power versus the probe pulse width are plotted in Figure 3b. From Figure 3a, it is obvious that the BGS spectrum becomes narrower and higher as the pulse width increases. The spectrum width of $H_{\mathrm{B}}(f, N)$, denoted as $W_{\mathrm{BGS}}$, decreases from $107.9 \mathrm{MHz}$ to $87.15 \mathrm{MHz}$. The relative peak power of $H_{\mathrm{B}}(f, \mathrm{~N})$, denoted as $P_{\mathrm{rp}}$, increases as the pulse width is increased from $12 \mathrm{~ns}$ to $52 \mathrm{~ns}$. 


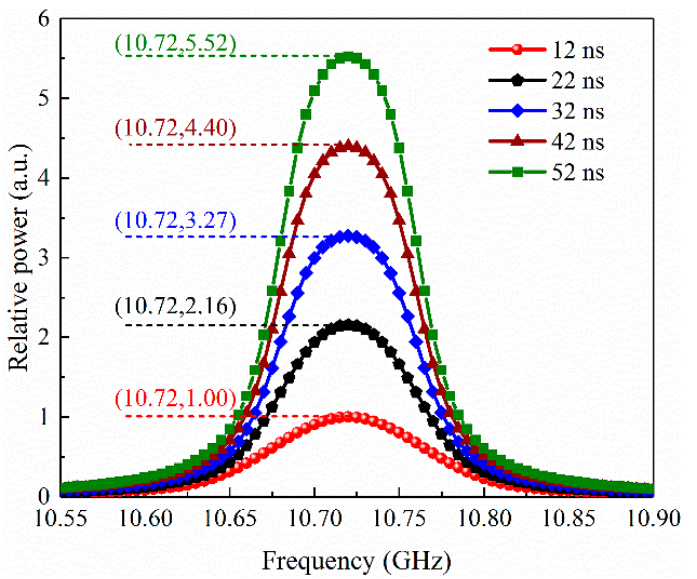

(a)

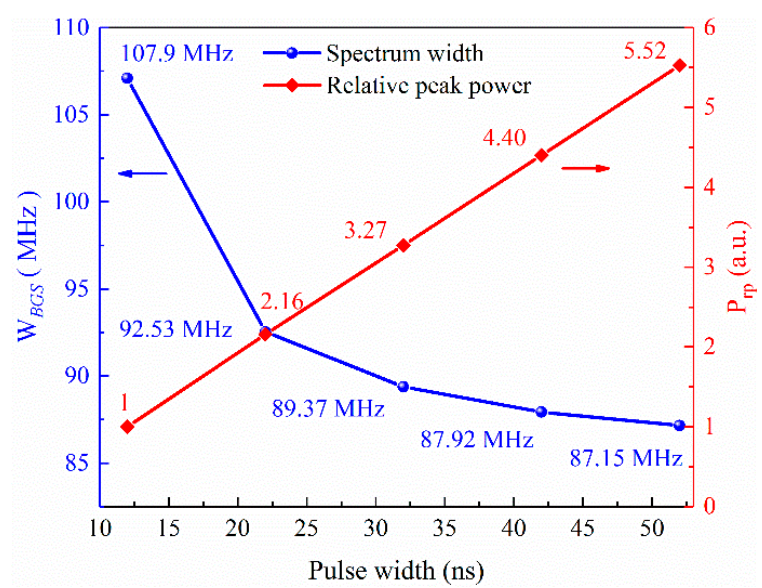

(b)

Figure 3. Numerical simulation results: (a) Profiles of $H_{\mathrm{B}}(f, N)$ under different probe pulse widths; (b) spectrum width and relative power versus probe pulse width.

Following simulation analysis of the BGS broadening, the relationship between the system SNR and the laser linewidth should be emphatically discussed. The photocurrent in coherent detection can be expressed as Equation (8) neglecting the polarization mismatch [28]:

$$
i(t, \Delta \varphi)=G R \sqrt{P_{\mathrm{SpB}} \cdot P_{\mathrm{Rf}}} \cdot \cos \left(2 \pi f_{\mathrm{B}} t+\Delta \varphi\right)
$$

where $G$ and $R$ are, respectively, the gain and responsivity of the detector, $P_{\mathrm{SpB}}(t)$ and $P_{\mathrm{Rf}}$ are the power of the spontaneous Brillouin backscattering and the reference light, respectively, $f_{\mathrm{B}}$ is the BFS, and $\Delta \varphi$ is the phase difference.

Because the coherent detection is highly phase sensitive, the phase fluctuation caused by $\Delta \varphi$ will greatly decrease the SNR and even lead to the central frequency jitter [25]. It has been proven that the phase fluctuation in the coherent detection can be viewed as a nonstationary random process and follows normal distribution with the mean value of $\mu$ and the variance of $\sigma^{2}$, given by Equation (9) [29]:

$$
\Delta \varphi \sim N\left(\mu, \sigma^{2}\right), \mu=0, \sigma^{2}=2 \pi n \Delta f \cdot \Delta L / c
$$

where $n$ is the refractive index of optical fiber, $c$ is the light speed in vacuum, $\Delta f$ is the laser linewidth, and $\Delta L$ is the optical path difference between the probe beam and the reference beam. Figure 4 gives a histogram of $\Delta \varphi$ utilized in the simulation when $\Delta f=1.05 \mathrm{MHz}, \Delta L=20 \mathrm{~km}$.

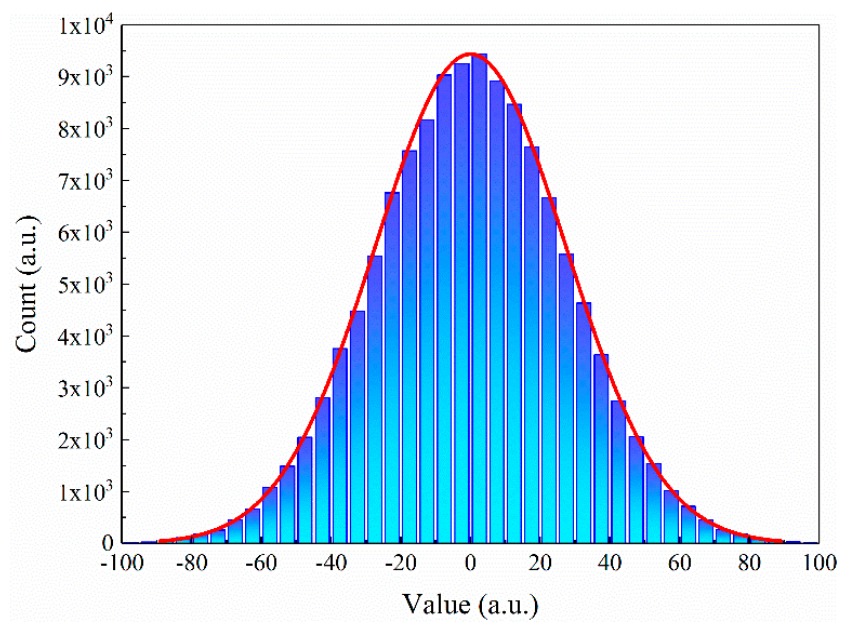

Figure 4. Histogram of $\Delta \varphi$ utilized in the simulation when $\Delta f=1.05 \mathrm{MHz}$ and $\Delta L=20 \mathrm{~km}$. 
To analyze the relationship between SNR and $\Delta \varphi$ succinctly, we treated phase fluctuation as a major noise contribution, regardless of the thermal noise and shot noise. The power of useful signal can be expressed as:

$$
<i_{S}^{2}(t)>=\frac{1}{\tau} \int_{0}^{\tau} i^{2}(t, \Delta \varphi) d t
$$

The power of noise can be expressed as:

$$
<i_{\mathrm{N}}^{2}(t)>=\frac{\sum_{k=1}^{N_{\mathrm{t}}}[i(k \Delta t, \Delta \varphi)-i(k \Delta t, 0)]^{2}}{N_{\mathrm{t}}-1}
$$

where $\Delta t$ is the sampling interval of $i(t, \Delta \varphi)$, and $N_{\mathrm{t}}=\tau / \Delta t$ is the number of samples. Hence, the SNR can be given by Equation (12):

$$
S N R=P_{\mathrm{rp}}(\tau) \cdot \frac{<i_{\mathrm{S}}^{2}(t)>}{<i_{\mathrm{N}}^{2}(t)>}=\frac{P_{\mathrm{rp}}(\tau) \frac{1}{\tau} \int_{0}^{\tau} i^{2}(t, \Delta \varphi) d t}{\frac{\sum_{k=1}^{N_{\mathrm{t}}}[i(k \Delta t, \Delta \varphi)-i(k \Delta t, 0)]^{2}}{\left(N_{\mathrm{t}}-1\right)}}
$$

where $P_{\operatorname{rp}}(\tau)$ is the relative peak power of the measured BGS closely related with the width of the probe pulse. The relative SNR versus laser linewidth under different pulse widths was numerically simulated and is plotted in Figure 5a according to Equation (12). Further, the BFS measurement error was calculated based on Equation (1) and normalized as shown in Figure 5b.

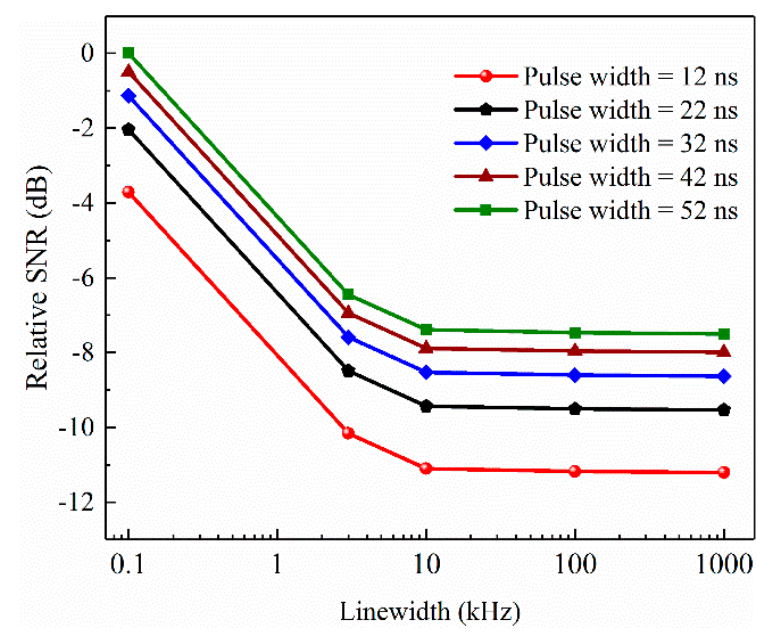

(a) Relative SNR

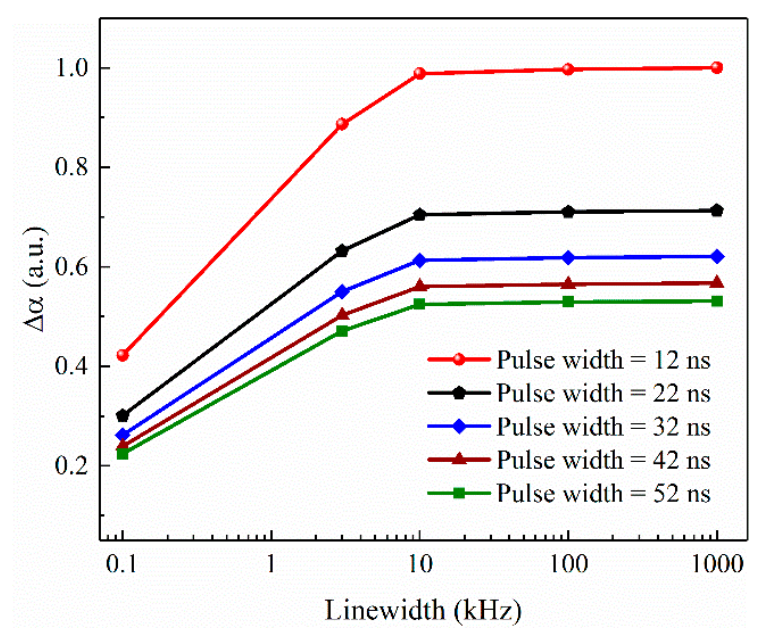

(b) BFS error

Figure 5. Numerical simulation results versus laser linewidth under different pulse widths: (a). Relative signal-to-noise ratio (SNR); (b). BFS accuracy. Model parameters utilized for simulations are listed in Table A1.

From Figure 5, it is obvious that the SNR is improved as the pulse width increases and the laser linewidth narrows. Under the same pulse width, the BFS error, $\Delta \alpha$, increases sharply as the laser linewidth broadens from $0.1 \mathrm{kHz}$ to $10 \mathrm{kHz}$, and grows slowly when the laser linewidth exceeds $10 \mathrm{kHz}$. Based on the above numerical simulations, it is theoretically proven that the BFS accuracy of BOTDR is improved as the laser width narrows and the pulse width increases. 


\section{Experiment Setup}

\subsection{Experiment Setup for Measuring Laser Linewidth}

To measure the linewidth of the utilized lasers, a linewidth measurement setup based on the delayed self-heterodyne interferometer (DSHI) was configured as shown in Figure 6.



Figure 6. Delayed self-heterodyne interferometer to measure the laser linewidth.

The laser output was split into two beams by a $1 \times 2$ coupler. The upper beam was delayed by a section of single-mode fiber. A polarization controller was used for reducing the polarization mismatch. The lower beam passes through an acoustic optical modulator (AOM) to be frequency shifted by $200 \mathrm{MHz}$. Finally, the two beams beat in a photodetector through a 3-dB coupler. The electronic signal after PD was acquired and recorded by an electrical spectrum analyzer (ESA). Five lasers with different linewidths were utilized in the experiments. Table 1 gives their nominal specifications.

Table 1. Nominal specifications of the five lasers used in the experiments.

\begin{tabular}{|c|c|c|c|}
\hline & Model & DFB-LSM-1550-20-PM & Manufacturer \\
\hline & Wavelength & $1550.12 \mathrm{~nm}$ & \multirow{3}{*}{ OPE $A K$} \\
\hline & Nominal linewidth & $1.1 \mathrm{MHz}$ & \\
\hline & Max power & $20 \mathrm{mw}$ & \\
\hline & Model & KG-DFB-15-M-10-S-FP & Manufacturer \\
\hline & Wavelength & $1550.12 \mathrm{~nm}$ & \multirow{3}{*}{$\begin{array}{l}\text { CONOUER } \\
\text { 康冠茪电 }\end{array}$} \\
\hline & Nominal linewidth & $100 \mathrm{kHz}$ & \\
\hline & Max power & $20 \mathrm{mw}$ & \\
\hline & Model & COSF-SC-1550-M & Manufacturer \\
\hline & Wavelength & $1550.12 \mathrm{~nm}$ & \multirow{3}{*}{ Ecompet } \\
\hline & Nominal linewidth & $10 \mathrm{kHz}$ & \\
\hline & Max power & $10 \mathrm{mw}$ & \\
\hline \multirow{8}{*}{ 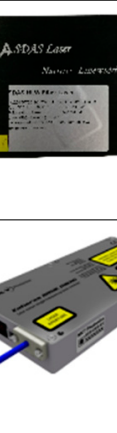 } & Model & SDAS-NLW-PL & Manufacturer \\
\hline & Wavelength & $1550.12 \mathrm{~nm}$ & \\
\hline & Nominal linewidth & $3 \mathrm{kHz}$ & \\
\hline & Max power & $17 \mathrm{mw}$ & \\
\hline & Model & Koheras BasiK E15 & Manufacturer \\
\hline & Wavelength & $1550.12 \mathrm{~nm}$ & \multirow{3}{*}{ N KT Thotonics } \\
\hline & Nominal linewidth & $<100 \mathrm{~Hz}$ & \\
\hline & Max power & $40 \mathrm{mw}$ & \\
\hline
\end{tabular}




\subsection{FS-BOTDR Setup for Measuring BFS}

A FS-BOTDR for measuring BFS change arising from temperature was built to verify the influence of the laser linewidth on BFS measurement accuracy, as shown in Figure 7.

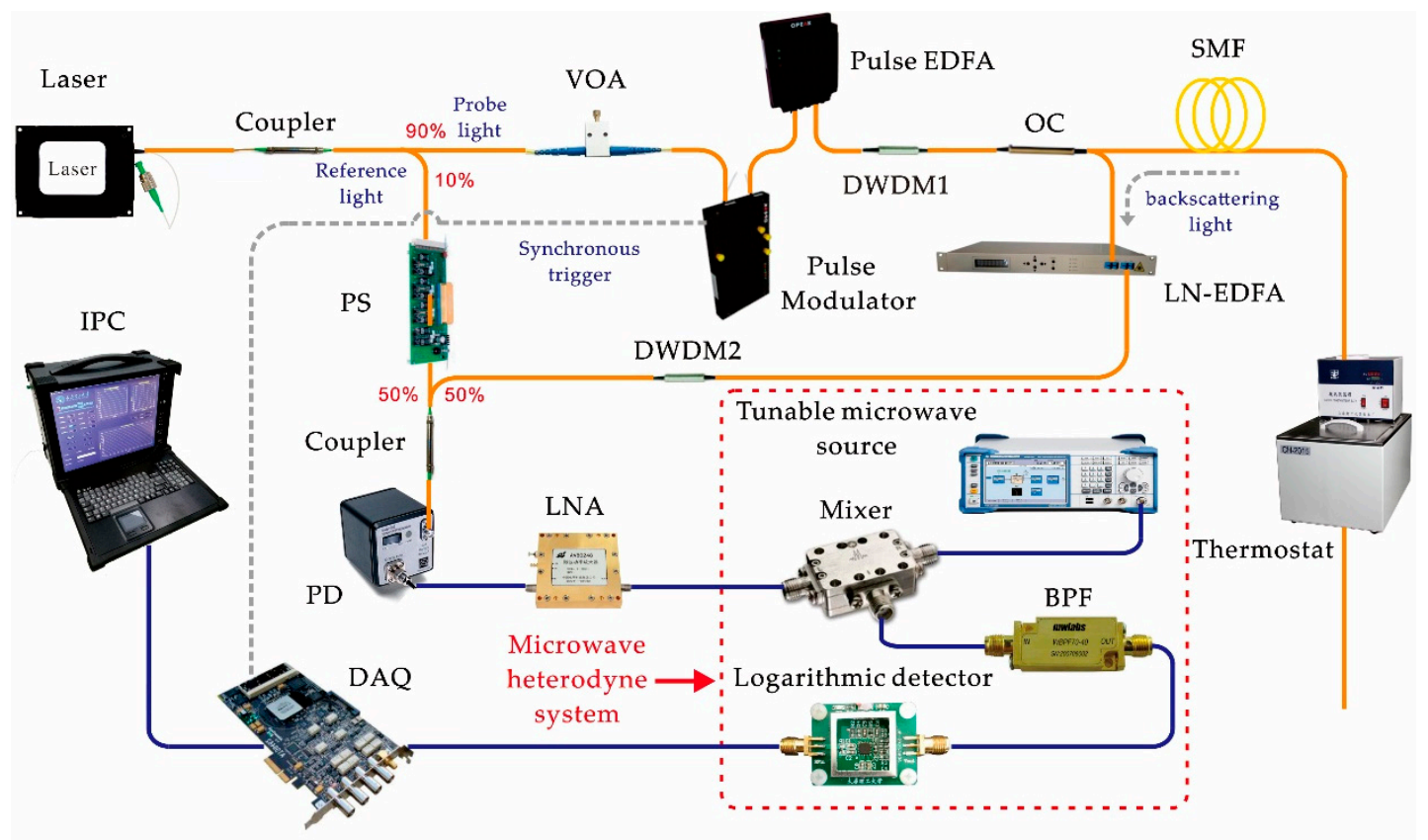

Figure 7. FS-BOTDR setup for temperature sensing.

The continuous light from the laser was divided into two beams through a 90:10 coupler. The $10 \%$ beam as reference light beats with the Brillouin backscattering for coherent heterodyne detection. The polarization scrambler (PS) was for eliminating the polarization noise. The $90 \%$ beam was attenuated to the proper power via a variable optical attenuator (VOA) and injected into a pulse modulator for generating probe pulses. The peak power of the probe pulse was amplified by a pulse erbium-doped fiber amplifier (Pulse EDFA) and filtered by a dense wavelength division multiplexer (DWDM1) to remove the amplified spontaneous emission (ASE) noise. Then, the probe pulse was launched into the sensing fiber through an optical circulator (OC). The Brillouin backscattering was amplified by the low-noise EDFA (LN-EDFA), filtered by the DWDM2, and then beats with the reference light in a 13.5-GHz photodetector (PD) through a 50:50 coupler. The beating signal was amplified by an electronic low-noise amplifier (LNA) and connected to a microwave heterodyne system.

In the microwave heterodyne system, the beating signal was mixed with a tunable microwave source to perform frequency scanning, the output frequency of which increased from $11.2 \mathrm{GHz}$ to $11.4 \mathrm{GHz}$ with a fixed frequency internal. The mixed signal was filtered by a BPF with the center frequency of $600 \mathrm{MHz}$ and bandwidth of $87 \mathrm{MHz}$. The time-domain power trace along the sensing fiber at every scanned frequency obtained by a logarithmic detector and acquired by the data acquisition (DAQ) digitalizer. Every power trace was averaged $2^{13}$ times. Eventually, the power points obtained at all scanned frequencies were fitted to the Lorentzian profile in an industrial personal computer (IPC), for calculating the BFS distribution along the fiber.

\section{Experiment Results and Discussions}

\subsection{Measurement of Laser Linewidth}

The length of the delay fiber can be settled to $25 \mathrm{~km}$ for measuring the nominal linewidth from $3 \mathrm{kHz}$ to $1.0 \mathrm{MHz}$ [30], and can be settled to $2950 \mathrm{~m}$ for less than $100 \mathrm{~Hz}$ [31]. The beating spectrums and fitting results are shown in Figure 8. In Figure 8a-d, the FWHM of the fitted spectrum was twice 
the laser linewidth, hence the measured linewidths of five lasers were, respectively, $1.05 \mathrm{MHz}, 101 \mathrm{kHz}$, $10.2 \mathrm{kHz}$, and $3.1 \mathrm{k} \mathrm{Hz}$, which were close to the nominal specifications. For the linewidth less than $100 \mathrm{~Hz}$, the accurate measured linewidth was calculated by utilizing the value of $\Delta S$ by the amplitude difference comparison of coherent envelope (ADCCE) method (See Appendix B) [31], because the length of the $2950 \mathrm{~m}$ delay fiber was much shorter than the coherence length of the measured laser. The accurate linewidth was $98 \mathrm{~Hz}$, as shown in Figure 8e, according to the ADCCE method.

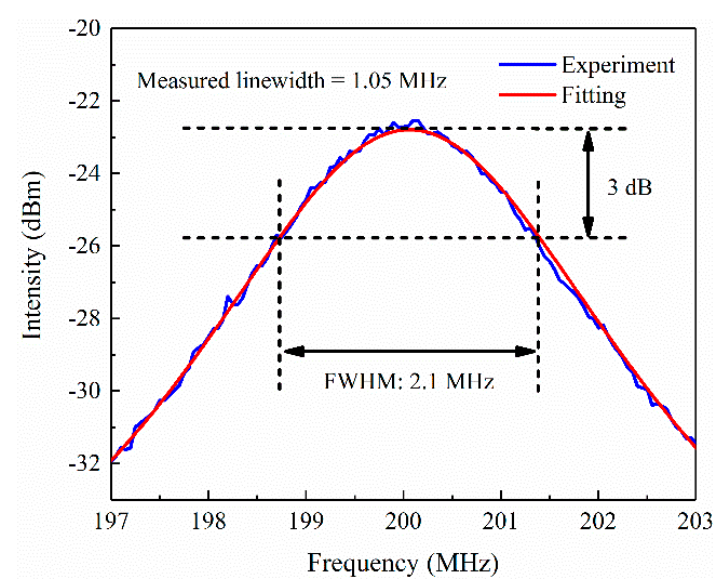

(a) Nominal linewidth $=1.1 \mathrm{MHz}$

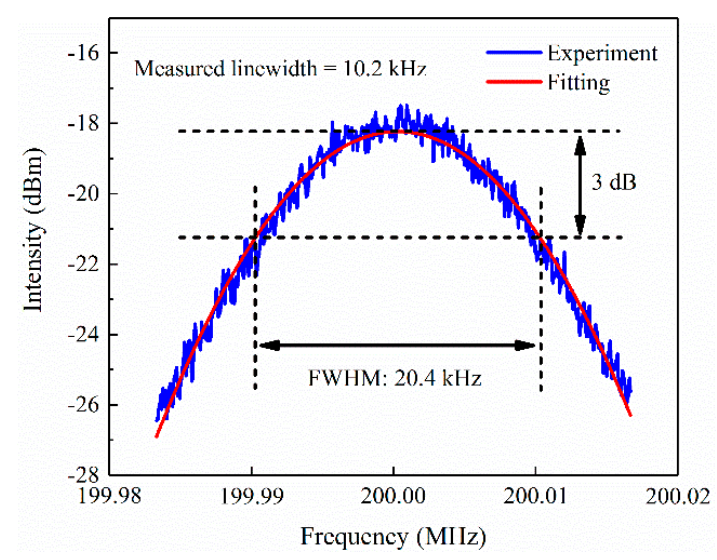

(c) Nominal linewidth $=10 \mathrm{kHz}$

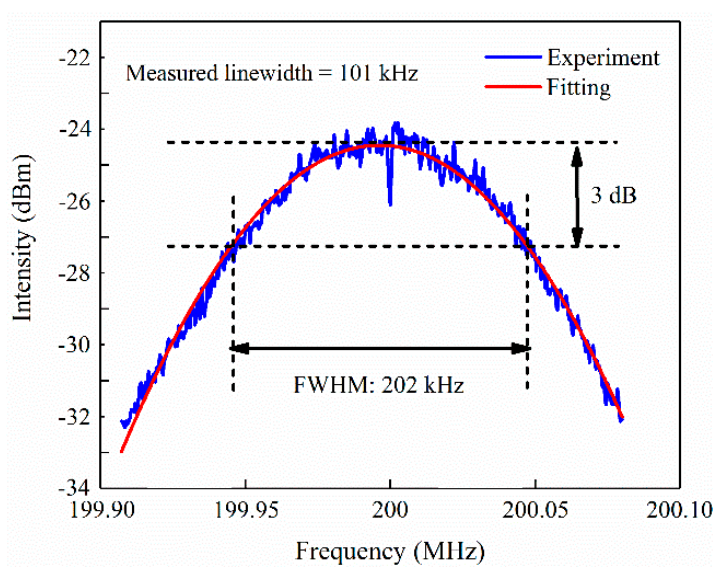

(b) Nominal linewidth $=100 \mathrm{kHz}$

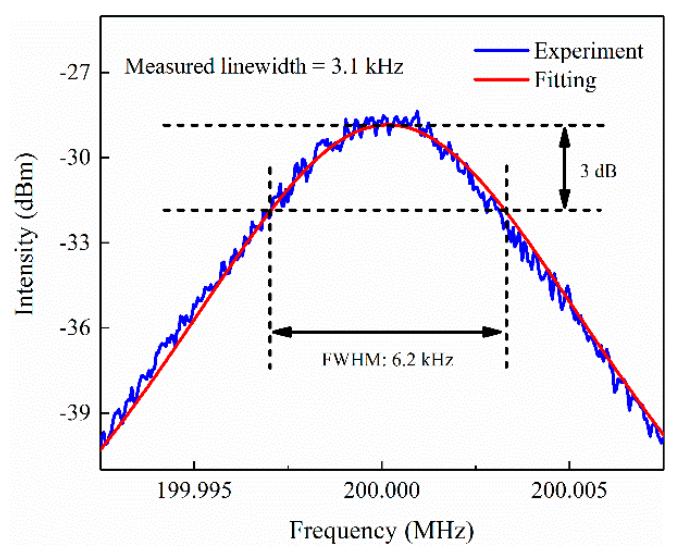

(d) Nominal linewidth $=3 \mathrm{kHz}$

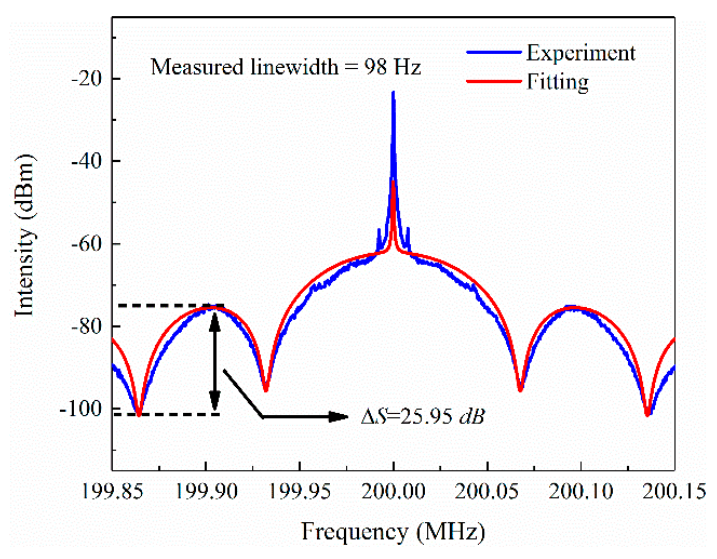

(e) Nominal linewidth $<100 \mathrm{~Hz}$

Figure 8. Linewidth measurement results of five lasers, of which the nominal linewidth are respectively: (a). $1.1 \mathrm{MHz}$; (b). $100 \mathrm{kHz}$; (c). $10 \mathrm{kHz}$; (d). $3 \mathrm{kHz}$; (e). $<100 \mathrm{~Hz}$. 


\subsection{BFS Distribution Measurement}

To verify whether the built FS-BOTDR is capable of measuring the BFS change normally, the temperature measurements were firstly performed utilizing the five lasers mentioned above as the seed source, respectively. Figure 9 gives the configuration of the tested fiber for temperature measurement. The fiber 1 with the length of $9865 \mathrm{~m}$ and the fiber 3 with the length of $305 \mathrm{~m}$ were placed at room temperature of $\sim 25^{\circ} \mathrm{C}$. The fiber 2 with the length of $30 \mathrm{~m}$ was placed in a thermostat, the temperature of which was adjusted to change the BFS of fiber 2 . As a contrast, the BFS of fiber 1 and fiber 3 were kept nearly constant in the room temperature.

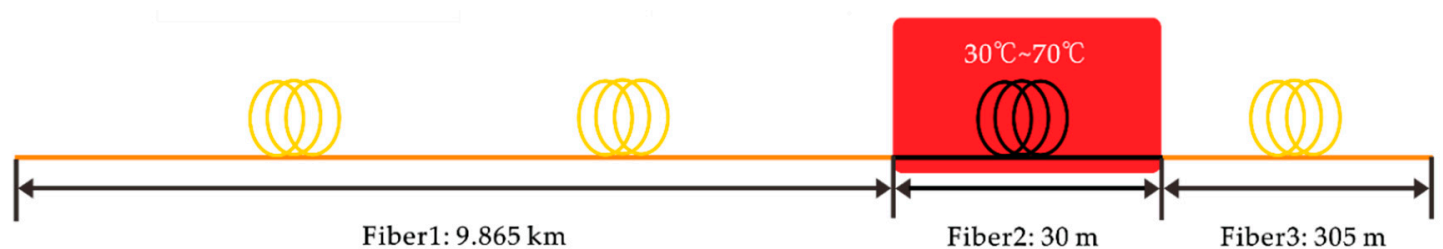

Figure 9. Configuration of the tested fiber for temperature measurement.

During the experiments, the width of the probe pulse light was set to $42 \mathrm{~ns}$ and the peak power was set to $23.09 \mathrm{dBm}$. The thermostat temperature was sequentially adjusted to $30{ }^{\circ} \mathrm{C}, 40{ }^{\circ} \mathrm{C}, 50{ }^{\circ} \mathrm{C}, 60{ }^{\circ} \mathrm{C}$, and $70^{\circ} \mathrm{C}$, respectively. Then, the BFS distribution measured by five lasers at different temperatures are shown in Figure 10a-e.

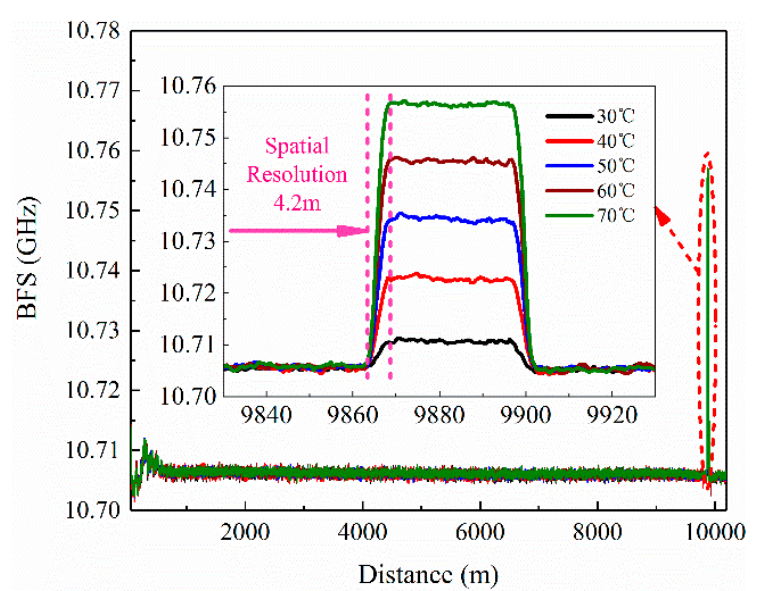

(a) Laser linewidth $=1.05 \mathrm{MHz}$

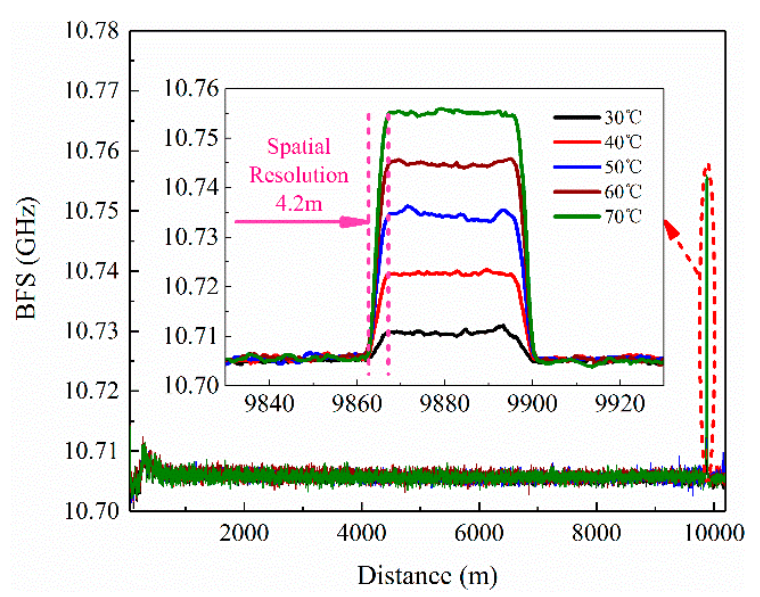

(c) Laser linewidth $=10.2 \mathrm{kHz}$

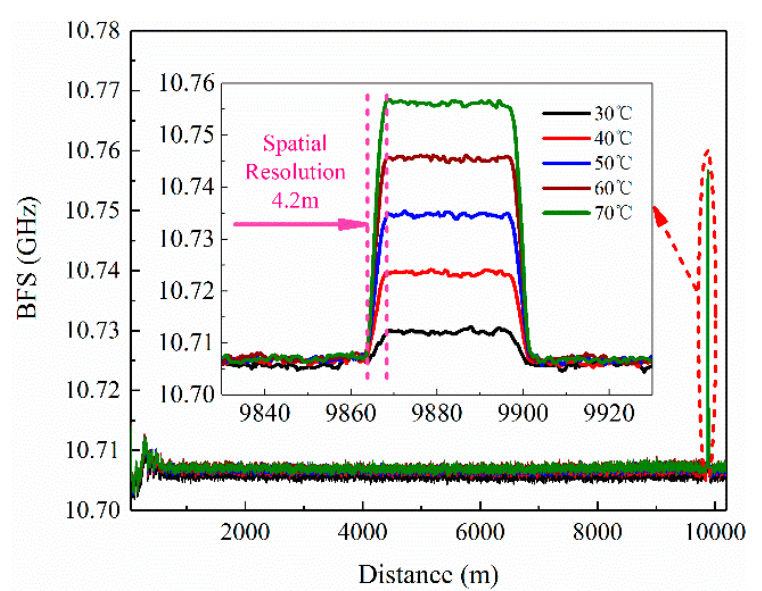

(b) Laser linewidth $=101 \mathrm{kHz}$

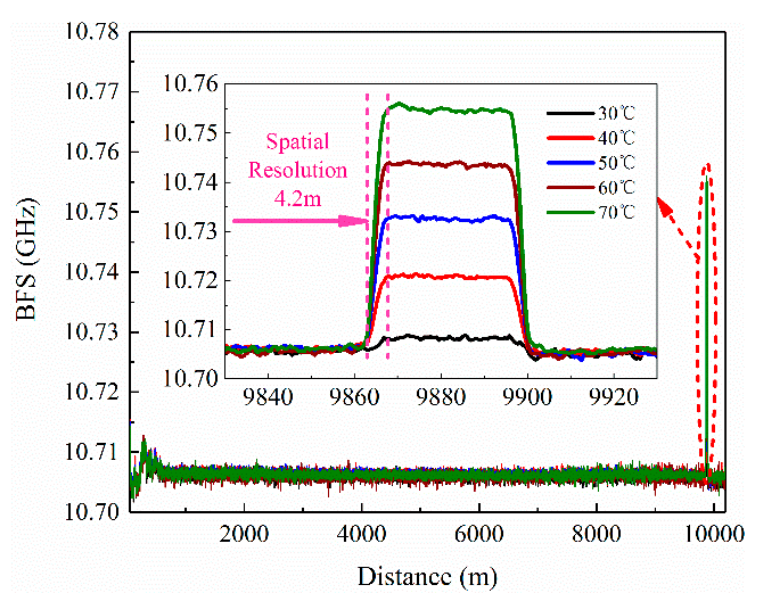

(d) Laser linewidth $=3.1 \mathrm{kHz}$

Figure 10. Cont. 


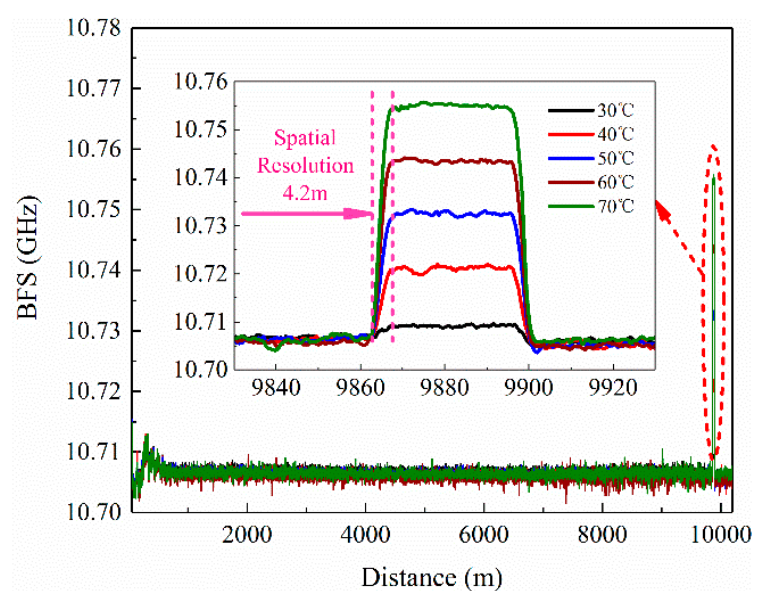

(e) Laser linewidth $=98 \mathrm{~Hz}$

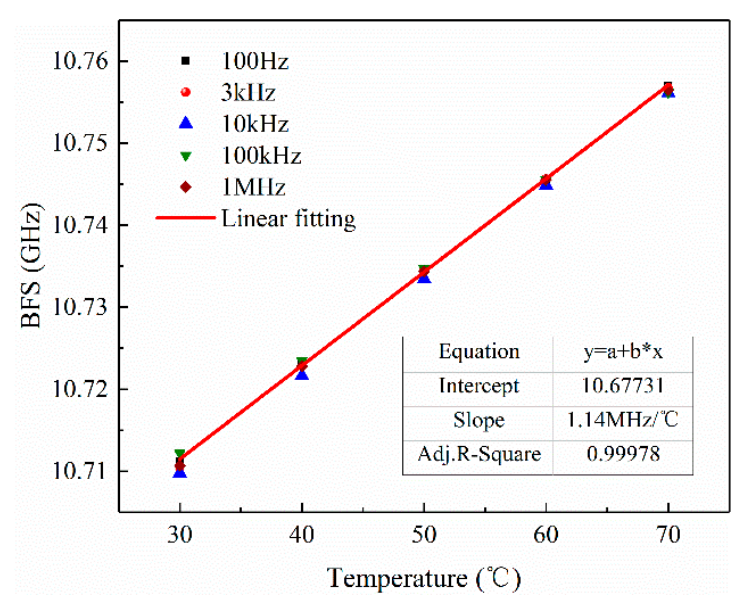

(f) Measured results of BFS versus temperature

Figure 10. BFS distributions at different temperatures when the laser linewidth is: (a) $1.05 \mathrm{MHz}$; (b) $101 \mathrm{kHz}$; (c) $10.2 \mathrm{kHz}$; (d) $3.1 \mathrm{kHz}$; and (e) $98 \mathrm{~Hz}$. (f) Measured results of BFS versus temperature.

From Figure 10a-e, it can be clearly seen that all obtained BFS for fiber 2, which was placed in the thermostat, increased linearly as the temperature increased from $30^{\circ} \mathrm{C}$ to $70^{\circ} \mathrm{C}$. As a contrast, the BFS for fiber 1 and fiber 3, which stayed in the room temperature of $25^{\circ} \mathrm{C}$, remained almost constant. Besides, the measured spatial resolution was $4.2 \mathrm{~m}$, which conformed to the pulse width of $42 \mathrm{~ns}$. Figure $10 \mathrm{f}$ plots the measured results of BFS versus temperature in detail, and the linear fitting was performed based on the measured data. It shows that the slope coefficient between the temperature and the BFS was $1.14 \mathrm{MHz} /{ }^{\circ} \mathrm{C}$, neatly coincided with that we reported previously [32]. The adjusted $\mathrm{R}$-square was 0.99978 , which indicates a strong linear relationship.

From the above experimental results, it is proven that the built FS-BOTDR setup was capable of measuring the BFS change normally, although utilizing five linewidth-different lasers as the seed light source, respectively. In the following section, further experiments were taken based on this setup to evaluate the influence of the laser linewidth on the BGS spectrum and the BFS accuracy in detail.

\subsection{BGS Width Evaluation}

To verify the influence of the laser linewidth on the BGS width, multiple BFS measurements were taken by utilizing the five lasers mentioned above as the seed source, respectively, when the temperature of the thermostat was fixed at $50^{\circ} \mathrm{C}$. The room temperature was kept at a roughly constant temperature of $25^{\circ} \mathrm{C}$. The width of the probe pulse was orderly adjusted to $12 \mathrm{~ns}, 22 \mathrm{~ns}, 32 \mathrm{~ns}$, and $42 \mathrm{~ns}$, with the same peak power of $23.09 \mathrm{dBm}$. For further analysis, we extracted the measured BGSs at $9880 \mathrm{~m}$ of tested fiber, the middle of the heated fiber, and fitted them to the Lorentzian profile based on the Levenberg-Marquart algorithm. These BGSs were finally normalized by peak power and are presented in Figure 11a-e, where the relative frequency of the horizontal axis was obtained by $f-f_{\mathrm{B}}$. The BGS widths versus the laser linewidth is summarily plotted in Figure 11f, when the width of the probe pulse was increased from $12 \mathrm{~ns}$ to $42 \mathrm{~ns}$.

From Figure 11f, the BGS width narrowed from $\sim 108 \mathrm{MHz}$ to $\sim 88 \mathrm{MHz}$ as the probe pulse width was increased from $12 \mathrm{~ns}$ to $42 \mathrm{~ns}$, which roughly agrees with the theoretical simulations (see Figure $3 \mathrm{~b}$, blue line). However, the BGS width showed no distinct tendency as the laser width was broadened from $98 \mathrm{~Hz}$ to $1.05 \mathrm{MHz}$. Hence, it proves that the measured BGS width depended largely on the probe pulse width when the laser linewidth was less than $1 \mathrm{MHz}$, which conformed to the conclusion reported previously [23]. 


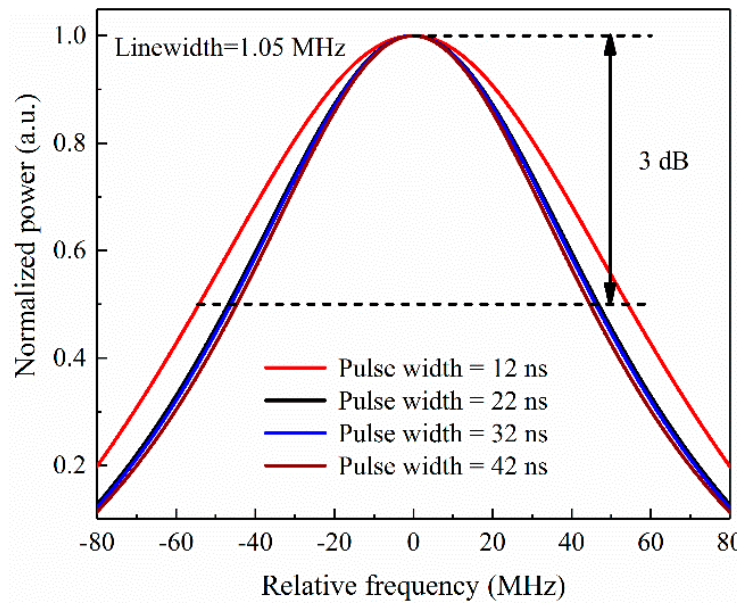

(a) Linewidth $=1.05 \mathrm{MHz}$

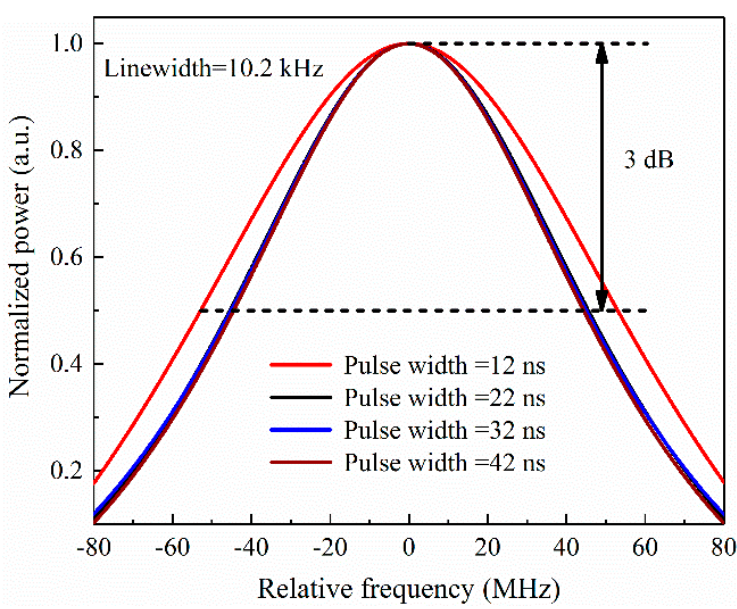

(c) Linewidth $=10.2 \mathrm{kHz}$

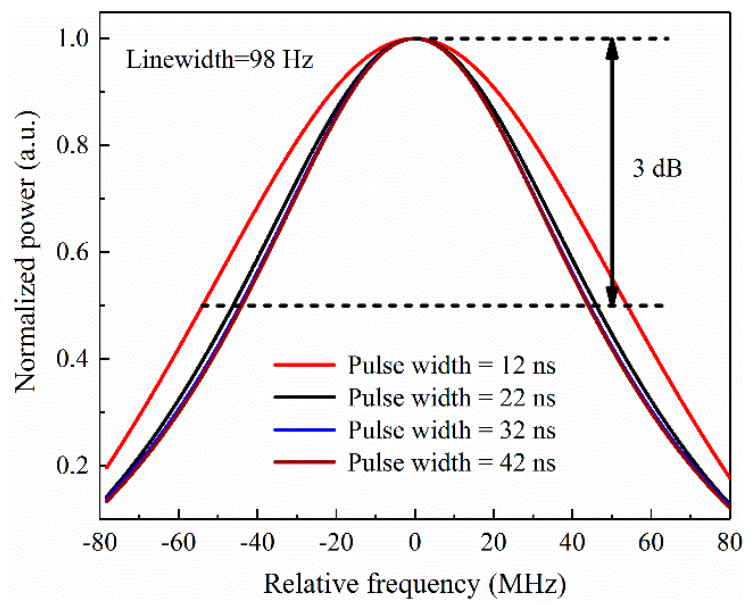

(e) Linewidth $=98 \mathrm{~Hz}$

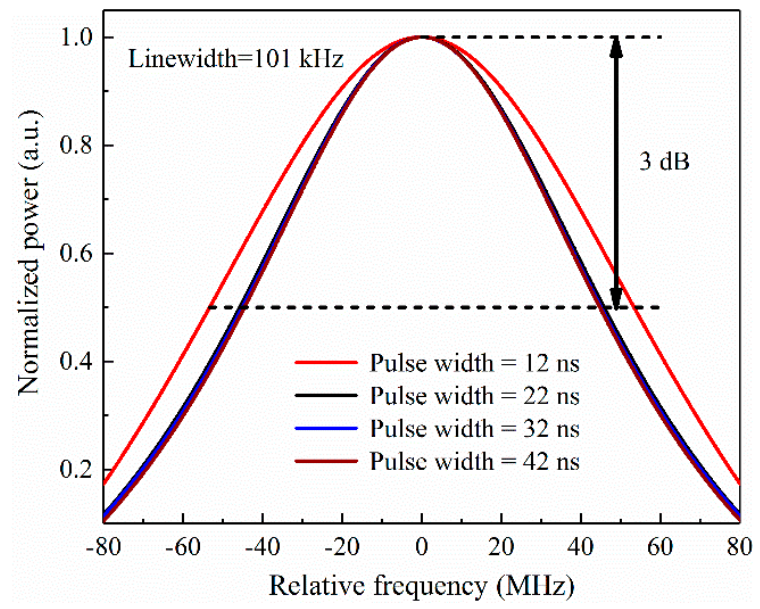

(b) Linewidth $=101 \mathrm{kHz}$

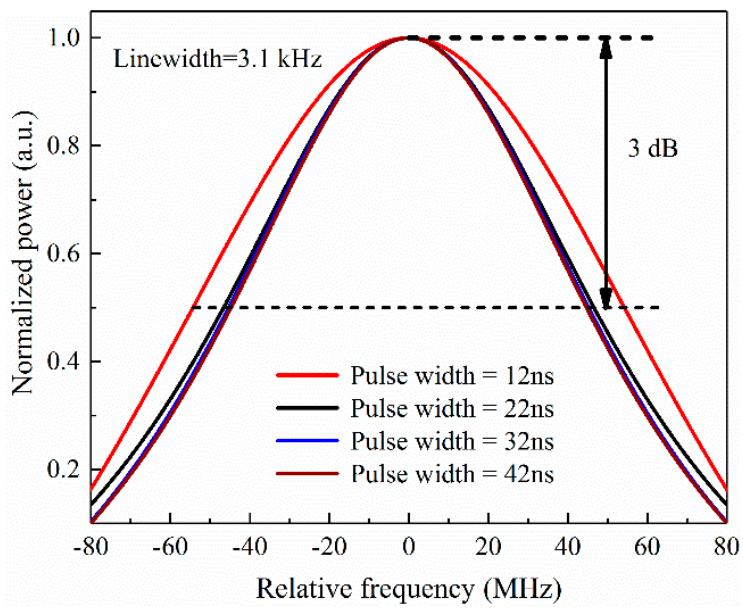

(d) Linewidth $=3.1 \mathrm{kHz}$

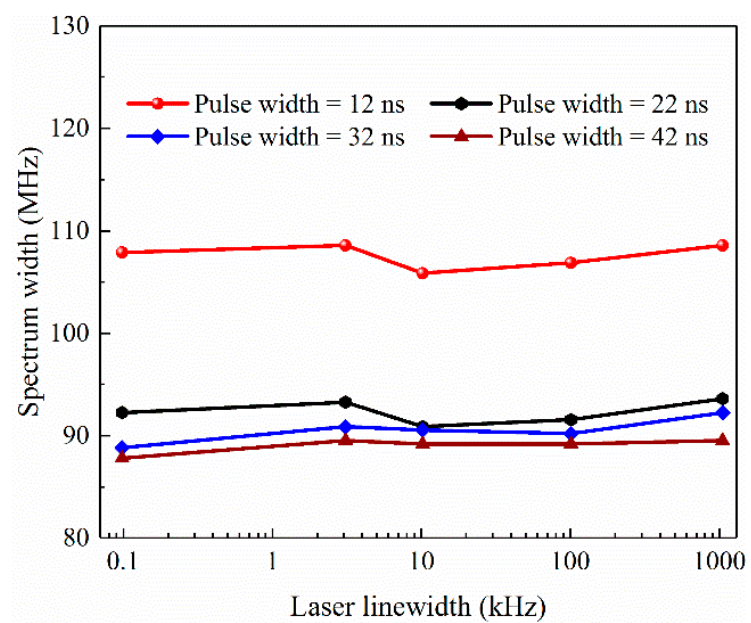

(f) BGS widths versus laser linewidth and pulse width

Figure 11. Normalized BGS extracted at the middle of the heated fiber when the laser linewidth is: (a) $1.05 \mathrm{MHz}$, (b) $101 \mathrm{kHz}$, (c) $10.2 \mathrm{kHz}$, (d) $3.1 \mathrm{kHz}$, and (e) $98 \mathrm{~Hz}$; (f) BGS widths versus laser linewidth and pulse width. 


\subsection{BFS Accuracy Evaluation}

For further evaluating the influence of the laser linewidth on the BFS accuracy, the measured BFS over the heated fiber were specially extracted and are shown in the inset of Figure 12a-e, eliminating the rise and fall edge of BFS change. The root-mean-square errors (RMSEs) of the extracted BFS distribution were calculated and plotted versus the laser linewidth and pulse width, as shown in Figure 12f.

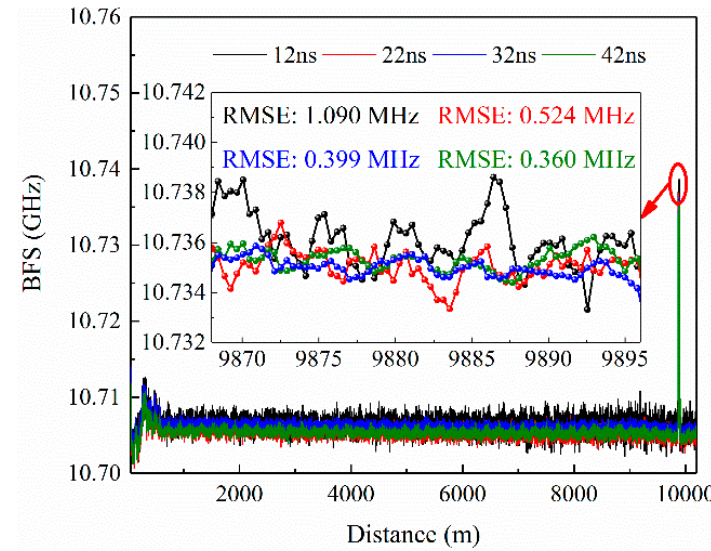

(a) Linewidth $=1.05 \mathrm{MHz}$



(c) Linewidth $=10.2 \mathrm{kHz}$

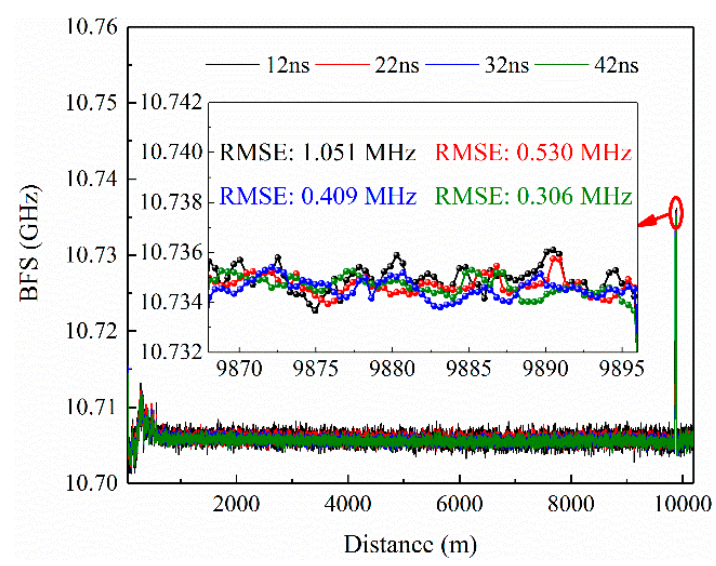

(e) Linewidth $=98 \mathrm{~Hz}$

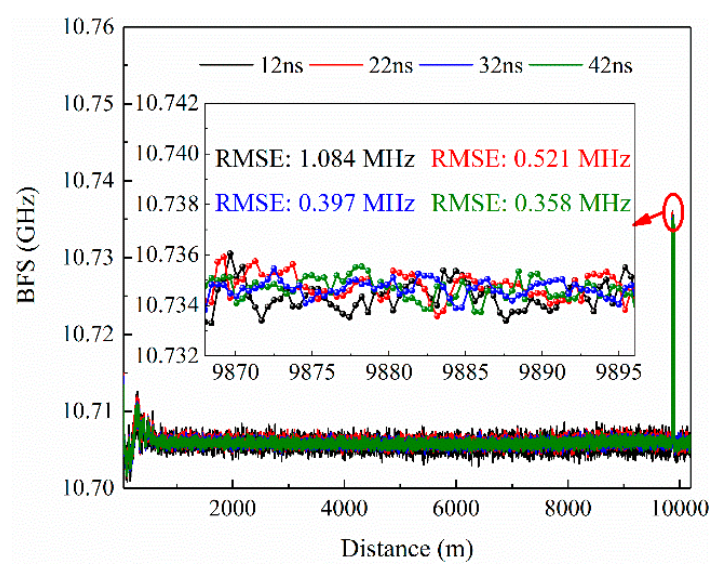

(b) Linewidth $=101 \mathrm{kHz}$



(d) Linewidth $=3.1 \mathrm{kHz}$



(f) BFS RMSEs versus laser linewidth and pulse width

Figure 12. Measured BFS distribution when the linewidth is: (a) $1.05 \mathrm{MHz}$, (b) $101 \mathrm{kHz}$, (c) $10.2 \mathrm{kHz}$, (d) $3.1 \mathrm{kHz}$, and (e) $98 \mathrm{~Hz}$; (f) BFS root-mean-square errors (RMSEs) versus laser linewidth and probe pulse width. 
From Figure 12f, it can be seen that the RMSE of BFS was related with both the laser linewidth and the probe pulse width. It decreased obviously when the pulse width was extended from $12 \mathrm{~ns}$ to $42 \mathrm{~ns}$. When the pulse width was fixed, the RMSE of BFS decreased with the laser linewidth narrowing from $1.05 \mathrm{MHz}$ to $3.1 \mathrm{kHz}$, which was in good agreement with the numerical simulations. However, it unexpectedly increased as the laser linewidth narrowed from $3.1 \mathrm{kHz}$ to $98 \mathrm{~Hz}$, which was inconsistent with the simulation results. We contribute this exception to the coherent Rayleigh noise (CRN). The CRN increases sharply as the laser linewidth becomes narrower [33]. Additionally, it has been proven that the CRN cannot be reduced by signal averaging [34]. Hence, the accuracy of BOTDR will decrease once the linewidth of the seed laser becomes narrow to a certain value, such as $98 \mathrm{~Hz}$ in this paper, because the extremely-narrow laser linewidth enhances the CRN largely and further results in sharp SNR deterioration of the BOTDR.

Based on the above analysis, it was demonstrated that the BFS accuracy improves when the laser linewidth narrows. However, the BFS accuracy will deteriorate when the laser linewidth is so narrow that the CRN is enhanced sharply. The measured results of BFS RMSE and BGS width ( $\left.\boldsymbol{W}_{\mathbf{B G S}}\right)$ by respectively utilizing five different-linewidth lasers as the seed source are summarily listed in Table 2, where the probe pulse width was $12 \mathrm{~ns}, 22 \mathrm{~ns}, 32 \mathrm{~ns}$, and $42 \mathrm{~ns}$ with the same peak power of $23.09 \mathrm{dBm}$.

Table 2. Summary list of measured results in experiments.

\begin{tabular}{cccccccccc}
\hline \multirow{2}{*}{$\begin{array}{c}\text { Laser } \\
\text { Linewidth }\end{array}$} & $\begin{array}{c}\text { Peak } \\
\text { Power }\end{array}$ & \multicolumn{2}{c}{ 12 ns } & \multicolumn{2}{c}{ 22 ns } & \multicolumn{2}{c}{ 32 ns } & \multicolumn{2}{c}{ 42ns } \\
\cline { 3 - 10 } & RMSE & $W_{\text {BGS }}$ & RMSE & $\boldsymbol{W}_{\text {BGS }}$ & RMSE & $\boldsymbol{W}_{\text {BGS }}$ & RMSE & $\boldsymbol{W}_{\text {BGS }}$ \\
\hline & $\mathbf{d B m}$ & $\mathbf{M H z}$ & $\mathbf{M H z}$ & $\mathbf{M H z}$ & $\mathbf{M H z}$ & $\mathbf{M H z}$ & $\mathbf{M H z}$ & $\mathbf{M H z}$ & $\mathbf{M H z}$ \\
\hline & 23.09 & 1.090 & 108.6 & 0.524 & 93.6 & 0.399 & 92.2 & 0.360 & 89.5 \\
$1.05 \mathrm{MHz}$ & 23.09 & 1.084 & 106.9 & 0.521 & 91.6 & 0.397 & 90.2 & 0.358 & 89.2 \\
$101 \mathrm{kHz}$ & 23.09 & 1.021 & 105.8 & 0.460 & 90.9 & 0.335 & 90.5 & 0.272 & 89.2 \\
$10.2 \mathrm{kHz}$ & 23.09 & 0.805 & 108.6 & 0.424 & 93.3 & 0.261 & 90.9 & 0.212 & 89.5 \\
$3.1 \mathrm{kHz}$ & 23.09 & 1.051 & 107.9 & 0.531 & 92.2 & 0.409 & 88.8 & 0.306 & 89.8 \\
$98 \mathrm{~Hz}$ & & & & & & & & & \\
\hline
\end{tabular}

\section{Conclusions}

In this paper, the influence of the laser linewidth on BFS accuracy in the FS-BOTDR was analyzed. The BGS broadening effect and the SNR of the FS-BOTDR was numerically simulated by taking phase fluctuation as the major noise contribution. The simulation results presented that the RMSE of BFS decreases when the laser linewidth narrows. In the experiments, we utilized five different lasers as the seed source, respectively, to measure the BFS change. The linewidth of the five lasers were, correspondingly, $1.05 \mathrm{MHz}, 101 \mathrm{kHz}, 10.2 \mathrm{kHz}, 3.1 \mathrm{kHz}$, and $98 \mathrm{~Hz}$. As the pulse width increased from $12 \mathrm{~ns}$ to $42 \mathrm{~ns}$, the BGS width and BFS accuracy were further evaluated and discussed in detail.

The experimental results indicate that the BFS accuracy improves with the laser linewidth narrowing. However, the BFS accuracy will deteriorate when the laser linewidth decreases to a certain value, such as $98 \mathrm{~Hz}$ in this paper. The exception may arise from the increasing CRN related closely with the narrowing linewidth. Therefore, how the CRN affects the BFS accuracy needs to be further theoretically explained and experimentally verified in future, if an extremely-narrow-linewidth laser is utilized in a FS-BOTDR. The results in this paper will be helpful to choose an appropriate laser for BOTDR, and provide potential techniques for improving the measurement accuracy of temperature or strain based BOTDR.

Author Contributions: B.J. proposed the idea; Q.B. performed the theoretical analysis and wrote the paper; M.Y. performed the temperature experiments; B.X. tested the laser linewidths; D.W. and Y.W. analyzed the data; H.Z., Y.G. and M.Z. revised the manuscript.

Funding: This research was funded in part by the National Natural Science Foundation of China (61805167), in part by the Coal-Bed Methane Joint Research Fund of Shanxi Province, China (2015012005 and 2016012011), and in part by the Social Development Project of Shanxi Province Key Research Plan (201703D321034). 
Acknowledgments: The 1.05-MHz-linewidth laser and the 3.1-kHz-linewidth laser used in the experiments were freely provided by the Tianjin Opeak Technology Co. Ltd and Laser Institute of Shandong Academy of Science (LISD) in China, respectively. They are very helpful to the experiments in this paper. The authors thank the two companies very much.

Conflicts of Interest: The authors declare no conflict of interest.

\section{Appendix A}

Table A1. The model parameters for numerical simulations.

\begin{tabular}{cccc}
\hline Parameter & Symbol & Value & Unit \\
\hline BGS width for continuous light & $w$ & 28 & $\mathrm{MHz}$ \\
Brillouin frequency shift & $f_{\mathrm{B}}$ & 10.7 & $\mathrm{GHz}$ \\
Startting frequecy for scanning & $f_{\mathrm{S}}$ & 10.4 & $\mathrm{GHz}$ \\
frequency & $f_{\text {step }}$ & 1 & $\mathrm{MHz}$ \\
Frequency step & $N$ & 671 & $\mathrm{MHz}$ \\
Number of frequency-scanning points & $B$ & 87 & $\mathrm{~m} / \mathrm{s}$ \\
Bandwidth of the BPF & $c$ & $3 \times 10^{8}$ & $\mathrm{~ns}$ \\
Speed of light in vacuum & $n$ & 1.5 & $\mathrm{~Hz}$ \\
Refractive index & $\tau$ & $12,22,32,42,52$ & $\mathrm{~km}$ \\
Pulse width & $\Delta f$ & $100,3000,10,000,100,000,1,050,000$ & 20 \\
Laser linewidth & $\Delta L$ & &
\end{tabular}

\section{Appendix B}

Appendix B mainly describes how the laser linewidth was measured with the delay fiber, the length of which was much shorter than the coherence length of the measured laser. It is well known that self-heterodyne interferometer (DSHI) is a frequently-used technology to measure laser linewidth. The output power spectrum of the DSHI can be expressed by $S(f, \Delta f)[31]$ :

$$
\begin{gathered}
S(f, \Delta f)=S_{1} S_{2} \\
S_{1}=\frac{P_{0}^{2}}{4 \pi} \frac{\Delta f^{2}}{\Delta f^{2}+\left(f-f_{1}\right)^{2}} \\
S_{2}=1-\exp \left(-2 \pi \Delta f \tau_{d}\right)\left[\cos \left[2 \pi\left(f-f_{1}\right) \tau_{d}\right]+\Delta f \frac{\sin \left[2 \pi\left(f-f_{1}\right) \tau_{d}\right]}{f-f_{1}}\right]
\end{gathered}
$$

where $f$ is the optical frequency, and $f_{1}=200 \mathrm{MHz}$ is the frequency shift of AOM. $\tau_{\mathrm{d}}=n L / c$ is the delay time caused by the delay fiber, $L=2950 \mathrm{~m}$ is the length of delay fiber, $c=3 \times 10^{8} \mathrm{~m} / \mathrm{s}$ is the speed of light in vacuum, $n=1.5$ is the refractive index, and $\Delta f=98 \mathrm{~Hz}$ is the laser linewidth. The normalized power spectra of $S(f, \Delta f), S_{1}$, and $S_{2}$ obtained by numerical simulation are plotted in Figure A1a.

It has been experimentally and theoretically proven that the value of the contrast difference between the second peaks and second troughs (CDSPST, denoted as $\Delta S$ ), as shown in Figure A1a, is related closely to the product of the measured laser linewidth and the length of delay fiber. The relationship curve is plotted in Figure A1b. 


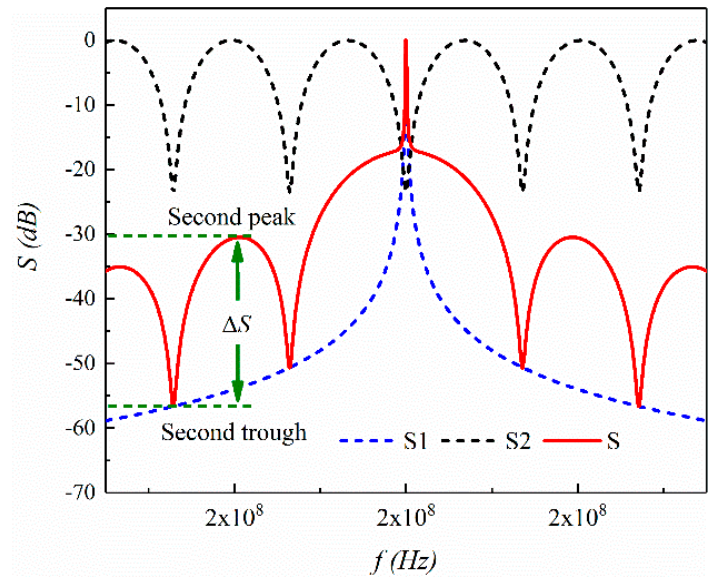

(a)

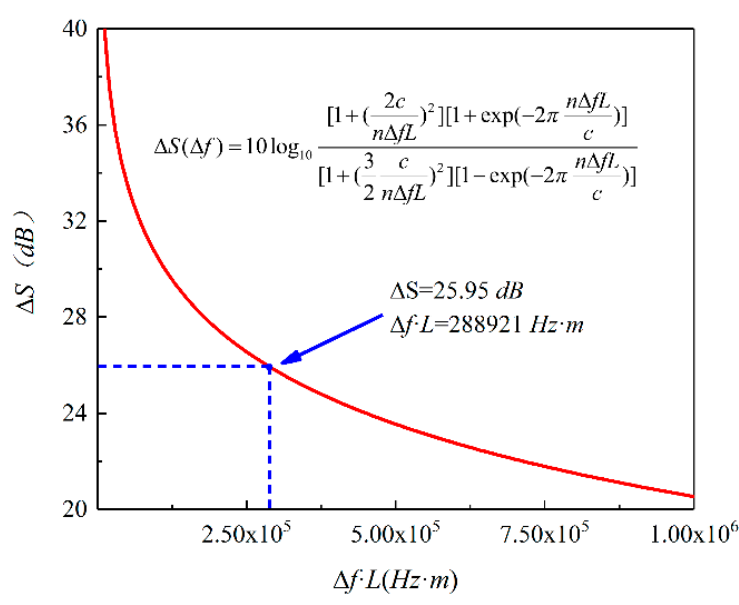

(b)

Figure A1. (a) Normalized power spectra of $S$; (b) relationship curve between $\Delta S$ and $\Delta f \cdot L$.

Hence, the value of $\Delta S$ can be used for calculating the measured laser linewidth once the length of the delay fiber is fixed. From Figure 8e, the obtained value of $\Delta S$ was $25.95 \mathrm{~dB}$, and the corresponding value of $\Delta f \cdot L$ was $288921 \mathrm{~Hz} \cdot \mathrm{m}$, as shown in Figure A1b.

Thus, the laser linewidth can be calculated according to the below expression:

$$
\Delta f=\frac{288921}{2950} \approx 98 \mathrm{~Hz}
$$

\section{References}

1. Kurashima, T.; Horiguchi, T.; Izumita, H.; Furukawa, S.; Koyamada, Y. Brillouin optical-fiber time domain reflectometry. IEICE Trans. Commun. 1993, E76-B, 382-390.

2. Geng, J.; Staines, S.; Blake, M.; Jiang, S. Distributed fiber temperature and strain sensor using coherent radio-frequency detection of spontaneous Brillouin scattering. Appl Opt. 2007, 46, 5928-5932. [CrossRef] [PubMed]

3. Li, A.; Wang, Y.; Hu, Q.; Che, D.; Chen, X.; Shieh, W. Measurement of distributed mode coupling in a few-mode fiber using a reconfigurable Brillouin OTDR. Opt. Lett. 2014, 39, 6418-6421. [CrossRef] [PubMed]

4. Hong, C.; Zhang, Y.; Li, G.; Zhang, M.; Liu, Z. Recent progress of using Brillouin distributed fiber optic sensors for geotechnical health monitoring. Sens. Actuators A 2017, 258, 131-145. [CrossRef]

5. Soto, M.A.; Bolognini, G.; Di Pasquale, F. Enhanced simultaneous distributed strain and temperature fiber sensor employing spontaneous Brillouin scattering and optical pulse coding. IEEE Photonic Technol. Lett. 2009, 21, 450-452. [CrossRef]

6. Kim, S.; Kwon, H.; Yang, I.; Lee, S.; Kim, J.; Kang, S. Performance of a distributed simultaneous strain and temperature sensor based on a Fabry-Perot laser diode and a dual-stage FBG optical demultiplexer. Sensors 2013, 13, 15452-15464. [CrossRef] [PubMed]

7. Webb, G.T.; Vardanega, P.J.; Hoult, N.A.; Fidler, P.R.A.; Bennett, P.J.; Middleton, C.R. Analysis of fiber-optic strain-monitoring data from a prestressed concrete bridge. J. Bridge Eng. 2017, 22, 05017002. [CrossRef]

8. Moffat, R.; Sotomayor, J.; Beltrán, J.F. Estimating tunnel wall displacements using a simple sensor based on a brillouin optical time domain reflectometer apparatus. Int. J. Rock Mech. Min. Sci. 2015, 75, 233-243. [CrossRef]

9. Hao, Y.; Cao, Y.; Ye, Q.; Cai, H.; Qu, R. On-line temperature monitoring in power transmission lines based on Brillouin optical time domain reflectometry. Optik 2015, 126, 2180-2183. [CrossRef]

10. Naruse, H.; Uehara, H.; Deguchi, T.; Fujihashi, K.; Onishi, M.; Espinoza, R.; Guzman, C.; Pardo, C.; Ortega, C.; Pinto, M. Application of a distributed fibre optic strain sensing system to monitoring changes in the state of an underground mine. Meas. Sci. Technol. 2007, 18, 3202-3210. [CrossRef] 
11. Maughan, S.M.; Kee, H.H.; Newson, T.P. 57-km single-ended spontaneous Brillouin-based distributed fiber temperature sensor using microwave coherent detection. Opt. Lett. 2001, 26, 331-333. [CrossRef] [PubMed]

12. Yao, Y.; Lu, Y.; Zhang, X.; Wang, F.; Wang, R. Reducing trade-off between spatial resolution and frequency accuracy in BOTDR using Cohen's Class signal processing method. IEEE Photonic Technol. Lett. 2012, 24, 1337-1339. [CrossRef]

13. Wang, F.; Zhan, W.; Lu, Y.; Yan, Z.; Zhang, X. Determining the change of Brillouin frequency shift by using the similarity matching method. J. Lightwave Technol. 2015, 33, 4101-4108. [CrossRef]

14. Zheng, H.; Fang, Z.; Wang, Z.; Lu, B.; Cao, Y.; Ye, Q.; Qu, R.; Cai, H. Brillouin frequency shift of fiber distributed sensors extracted from noisy signals by quadratic fitting. Sensors 2018, 18, 409. [CrossRef] [PubMed]

15. Hao, Y.; Ye, Q.; Pan, Z.; Cai, H.; Qu, R. Analysis of spontaneous Brillouin scattering spectrum for different modulated pulse shape. Optik 2013, 124, 2417-2420. [CrossRef]

16. Hao, Y.; Ye, Q.; Pan, Z.; Cai, H.; Qu, R.; Yang, Z. Effects of modulated pulse format on spontaneous Brillouin scattering spectrum and BOTDR sensing system. Opt. Laser Technol. 2013, 46, 37-41. [CrossRef]

17. Wang, F.; Zhu, C.; Cao, C.; Zhang, X. Enhancing the performance of BOTDR based on the combination of FFT technique and complementary coding. Opt. Express 2017, 25, 3504-3513. [CrossRef]

18. Hao, Y.; Ye, Q.; Pan, Z.; Cai, H.; Qu, R. Digital coherent detection research on Brillouin optical time domain reflectometry with simplex pulse codes. Chin. Phys. B 2014, 23. [CrossRef]

19. Lu, Y.; Yao, Y.; Zhao, X.; Wang, F.; Zhang, X. Influence of non-perfect extinction ratio of electro-optic modulator on signal-to-noise ratio of BOTDR. Opt. Commun. 2013, 297, 48-54. [CrossRef]

20. Zhang, Y.; Wu, X.; Ying, Z.; Zhang, X. Performance improvement for long-range BOTDR sensing system based on high extinction ratio modulator. Electron. Lett 2014, 50, 1014-1016. [CrossRef]

21. Lalam, N.; Ng, W.P.; Dai, X.; Wu, Q.; Fu, Y.Q. Performance improvement of Brillouin ring laser based BOTDR system employing a wavelength diversity technique. J. Lightwave Technol. 2018, 36, 1084-1090. [CrossRef]

22. Li, C.; Lu, Y.; Zhang, X.; Wang, F. SNR enhancement in Brillouin optical time domain reflectometer using multi-wavelength coherent detection. Electron. Lett 2012, 48, 1139-1141. [CrossRef]

23. Hao, Y.; Ye, Q.; Pan, Z.; Cai, H.; Qu, R. Influence of laser linewidth on performance of Brillouin optical time domain reflectometry. Chin. Phys. B 2013, 22. [CrossRef]

24. Naruse, H.; Tateda, M. Trade-off between the spatial and the frequency resolutions in measuring the power spectrum of the Brillouin backscattered light in an optical fiber. Appl. Opt. 1999, 38, 6516-6521. [CrossRef] [PubMed]

25. Zheng, Z.; Zhao, C.; Zhang, H.; Yang, S.; Zhang, D.; Yang, H.; Liu, J. Phase noise reduction by using dual-frequency laser in coherent detection. Opt. Laser Technol. 2016, 80, 169-175. [CrossRef]

26. Motil, A.; Bergman, A.; Tur, M. State of the art of Brillouin fiber-optic distributed sensing. Opt. Laser Technol. 2016, 78, 81-103. [CrossRef]

27. Nikles, M.; Thevenaz, L.; Robert, P.A. Brillouin gain spectrum characterization in single-mode optical fibers. J. Lightwave Technol. 1997, 15, 1842-1851. [CrossRef]

28. Hui, R.; O'Sullivan, M. Fiber Optic Measurement Techniques; Elsevier Academic Press: Burlington, MA, USA, 2009; pp. 196-199.

29. Du, Z.; Lu, L.; Wang, B.; Jiang, C. Effect of laser diode phase noise on coherent sensing systems. In Proceedings of the 13th International Conference on Advanced Laser Technologies, Tianjin, China, 3-6 September 2005. [CrossRef]

30. NKT Photonics. Laser Spectral Linewidth. Available online: http://www.sevensix.co.jp/wordpress/ wp-content/uploads/2017/07/Linewidth-Application-Note-Version-1-1-October-2013.pdf (accessed on 9 December 2018).

31. Huang, S.; Zhu, T.; Cao, Z.; Liu, M.; Deng, M.; Liu, J.; Li, X. Laser linewidth measurement based on amplitude difference comparison of coherent envelope. IEEE Photonic Technol. Lett. 2016, 28, 759-762. [CrossRef]

32. Liu, R.; Zhang, M.; Zhang, J.; Liu, Y.; Jin, B.; Bai, Q.; Li, Z. Temperature measurement accuracy enhancement in the Brillouin optical time domain reflectometry system using the sideband of Brillouin gain spectrum demodulation. Acta Phys. Sin. 2016, 65. [CrossRef] 
33. Reshak, A.H.; Shahimin, M.M.; Murad, S.A.Z.; Azizan, S. Simulation of Brillouin and Rayleigh scattering in distributed fibre optic for temperature and strain sensing application. Sens. Actuators A 2013, 190, 191-196. [CrossRef]

34. De Souza, K. Significance of coherent rayleigh noise in fibre-optic distributed temperature sensing based on spontaneous Brillouin scattering. Meas. Sci. Technol. 2006, 17, 1065-1069. [CrossRef] 